\begin{tabular}{|c|l|}
\hline Title & Fat solenoidal attractors \\
\hline Author(s) & Tsujii, M. \\
\hline Citation & Hokkaido University Preprint Series in Mathematics, 501, 1-20 \\
\hline Issue Date & 2000-11-1 \\
\hline DOI & 10.14943/83647 \\
\hline Doc URL & http://hdl.handle.net/2115/69251 \\
\hline Type & bulletin (article) \\
\hline File Information & pre501.pdf \\
\hline
\end{tabular}

Instructions for use 


\title{
Fat solenoidal attractors
}

\author{
Masato TSUJII
}

Series \#501. November 2000 


\section{HOKKAIDO UNIVERSITY PREPRINT SERIES IN MATHEMATICS}

\#477 T. Nakazi, On an invariant subspace whose common zero set is the zeros of some function, 11 pages. 1999.

\#478 M.-H. Giga and Y. Giga, Generalized motion by nonlocal curvature in the plane, 68 pages. 2000.

\#479 M.-H. Giga and Y. Giga, Crystalline and level set flow - Convergence of a crystalline algorithm for a general anisotropic curvature flow in the plane, 16 pages. 2000.

\#480 A. Arai and M. Hirokawa, Stability of ground states in sectors and its application to the Wigner-Weisskopf model, 16 pages. 2000.

\#481 T. Nakazi, Two dimensional $Q$-algebras, 11 pages. 2000.

\#482 N. H. Bingham and A. Inoue, Tauberian and Mercerian theorems for systems of kernels, 16 pages. 2000.

\#483 N. H. Bingham and A. Inoue, Abelian, Tauberian and Mercerian theorems for arithmetic sums, 29 pages. 2000.

\#484 I. A. Bogaevski and G. Ishikawa, Lagrange mappings of the first open Whitney umbrella, 22 pages. 2000.

\#485 A. Arai and H. Kawano, A class of deformations of the Schrödinger representation of the Heisenberg commutation relation and exact solution to a Heisenberg equation and a Schrödinger equation, 22 pages. 2000.

\#486 T. Nakazi, Functions in $N_{+}$with the positive real parts on the boundary, 21 pages. 2000.

\#487 Y. Shibukawa, Classification of the $R$-operator, 36 pages. 2000.

\#488 A. Inoue, Asymptotic behaviour for partial autocorrelation functions of fractional ARIMA processes, 20 pages. 2000.

\#489 S. Ohtani, Construction of unramified Galois extensions over maximal abelian extensions of algebraic number fields, 14 pages. 2000.

\#490 T. Nakazi and T. Yamamoto, The real part of an outer function and a Helson-Szegö weight, 13 pages. 2000 .

\#491 A. Yamagami, On Gouvêás conjecture on controlling the conductor, 11 pages. 2000.

\#492 I. Tsuda and M. Hatakeyama, Making sense of internal logic: Theory and a case study, 10 pages. 2000.

\#493 I. Tsuda, Towards an interpretation of dynamic neural activity in terms of chaotic dynamical systems, 73 pages. 2000.

\#494 T. Mikami, Optimal control for absolutely continuous stochastic processes and the mass transportation problem, 17 pages. 2000.

\#495 M. Arisawa and Y. Giga, Anisotropic curvature flow in a very thin domain, 21 pages. 2000.

\#496 T. Nakazi, Backward shift invariant subspaces in the bidisc, 9 pages. 2000.

\#497 Y. Giga, K. Inui, J. Kato and S. Matsui, Remarks on the uniqueness of bounded solutions of the NavierStokes equations, 4 pages. 2000.

\#498 Y. Giga, M. Paolini and P. Rybka, On the motion by singular interfacial energy, 21 pages. 2000.

\#499 J. Escher and Y. Giga, On a limiting motion and self-interactions of curves moved by the intermediate surface diffusion flow, 12 pages. 2000.

\#500 I. Tsuda and S. Kuroda, Cantor coding in the hippocampus, 20 pages. 2000. 


\title{
Fat solenoidal attractors
}

\author{
Masato TSUJII \\ Department of Mathematics \\ Hokkaido University
}

October 28,2000

\begin{abstract}
We study dynamical systems generated by skew products

$$
T: S^{1} \times \mathbb{R} \rightarrow S^{1} \times \mathbb{R}, \quad T(x, y)=(\ell x, \lambda y+f(x))
$$

where $\ell \geq 2,1 / \ell<\lambda<1$ and $f$ is a $C^{2}$ function on $S^{1}$. We show that the SBR measure for $T$ is absolutely continuous for almost every $f$.
\end{abstract}

\section{Introduction}

In this paper, we study a class of dynamical systems that stably admit an absolutely continuous ergodic measure (acem) with a negative Lyapunov exponent. It is well-known that expanding dynamical systems generally admit acem's whose Lyapunov exponents are all positive. The aim of this paper is to study another kind of acem's which is produced by a quite different mechanism: overlap and sliding in short.

We can find a typical example of such acem's in a paper of Alxander and Yorke[1], where the so-called generalized baker's transformation is considered:

$$
B:[-1,1] \times[-1,1] \circlearrowleft, \quad B(x, y)= \begin{cases}(2 x-1, \beta y+(1-\beta)) & x \geq 0 \\ (2 x+1, \beta y-(1-\beta)) & x<0\end{cases}
$$

When $\beta=1 / 2$, this map $B$ is nothing but the ordinary baker's transformation. Alxander and Yorke studied the case $1 / 2<\beta \leq 1$. In such case, the images of left and right halves of the domain, i.e., $B([-1,0] \times[-1,1])$ and $B([0,1] \times[-1,1])$ overlap with some sliding. This makes the dynamical nature of the map $B$ more complicated and interesting. They observed that the map $B$ admits an acem if and only if the number $\beta$ satisfies a delicate numerical condition: absolute continuity of the corresponding infinitely convoluted Bernoulli measure. As they noted, there are infinitely many numbers in $(1 / 2,1]($ e.g. $(\sqrt{5}-1) / 2)$ for which $B$ admits no acem's, according to a result of Erdös[2]. On the other hand, $B$ admits an acem for Lebesgue almost every $\beta$ in $(1 / 2,1]$ according to a more recent result of Solomyak[3]. 
In this paper, we consider a class of dynamical systems generated by maps

$$
T: S^{1} \times \mathbb{R} \rightarrow S^{1} \times \mathbb{R}, \quad T(x, y)=(\ell x, \lambda y+f(x))
$$

where $\ell \geq 2$ is an integer, $0<\lambda<1$ is a real number, and $f$ is a $C^{2}$ function on $S^{1}=\mathbb{R} / \mathbb{Z}$. We may regard this class of maps as a conceptual generalization of the generalized baker's transformations $B$ in the sense that the translation in vertical direction depends smoothly on $x$.

The map $T$ is a skew product on the expanding map $\tau: x \mapsto \ell x$ and it is uniformly contracting in the fiber direction. So $T$ is an Anosov endomorphism. The ergodic property of $T$ is rather simple: there exists an ergodic probability measure $\mu$ on $S^{1} \times \mathbb{R}$, for which Lebesgue almost every point $\mathbf{x} \in S^{1} \times \mathbb{R}$ is generic, that is,

$$
\lim _{n \rightarrow \infty} \frac{1}{n} \sum_{i=0}^{n-1} \delta_{T^{i}(\mathbf{x})}=\mu \quad \text { weakly. }
$$

(See section 2.) We will call this measure $\mu$ the $S B R$ measure for $T$.

The question is smoothness of the SBR measure $\mu$ with respect to the Lebesgue measure on $S^{1} \times \mathbb{R}$. In the case $\lambda \ell<1$, the SBR measure is totally singular because $T$ contracts area. The case $\lambda \ell>1$, which corresponds to the case $\beta>1 / 2$ for the generalized baker's transformations, is more interesting. We will focus on this case. First we give two examples in opposite directions. (The proof of the facts in these examples will be given in the last section. )

Example 1 Let $\ell=2,0.5<\lambda \leq 0.51$ and $f(x)=\sin 2 \pi x$. Then the $S B R$ measure $\mu$ for $T$ is absolutely continuous with respect to the Lebesgue measure of $S^{1} \times \mathbb{R}$. (See figure 1.)

Example 2 If $f(x)=\varphi(\tau(x))-\lambda \varphi(x)$ for some measurable function $\varphi$ on $S^{1}$, the SBR measure for $T$ is supported on the graph of $\varphi$ and totally singular.

We claim that the SBR measure is absolutely continuous for almost every $T$ and, moreover, that the absolute continuity is robust. Fix an integer $\ell \geq 2$. Let $\mathcal{D} \subset(0,1) \times C^{2}\left(S^{1}, \mathbb{R}\right)$ be the set of combinations $(\lambda, f)$ for which the SBR measure is absolutely continuous w.r.t. the Lebesgue measure on $S^{1} \times \mathbb{R}$. We consider the interior $\mathcal{D}^{\circ}$ of $\mathcal{D}$ with respect to the topology that is defined as the product of the canonical topology on $(0,1)$ and $C^{2}$-topology on $C^{2}\left(S^{1}, \mathbb{R}\right)$. The main result of this paper is the following.

Theorem 1 Let $\ell^{-1}<\lambda<1$. There exists a finite collection of $C^{\infty}$ functions $\varphi_{i}: S^{1} \rightarrow \mathbb{R}, i=1,2, \cdots, m$, such that, for any $C^{2}$ function $g \in C^{2}\left(S^{1}, \mathbb{R}\right)$, the subset of $\mathbb{R}^{m}$,

$$
\left\{\left(t_{1}, t_{2}, \cdots, t_{m}\right) \in \mathbb{R}^{m} \mid\left(\lambda, g(x)+\sum_{i=1}^{m} t_{i} \varphi_{i}(x)\right) \notin \mathcal{D}^{\circ}\right\},
$$

is a null set with respect to the Lebesgue mesure on $\mathbb{R}^{m}$. 


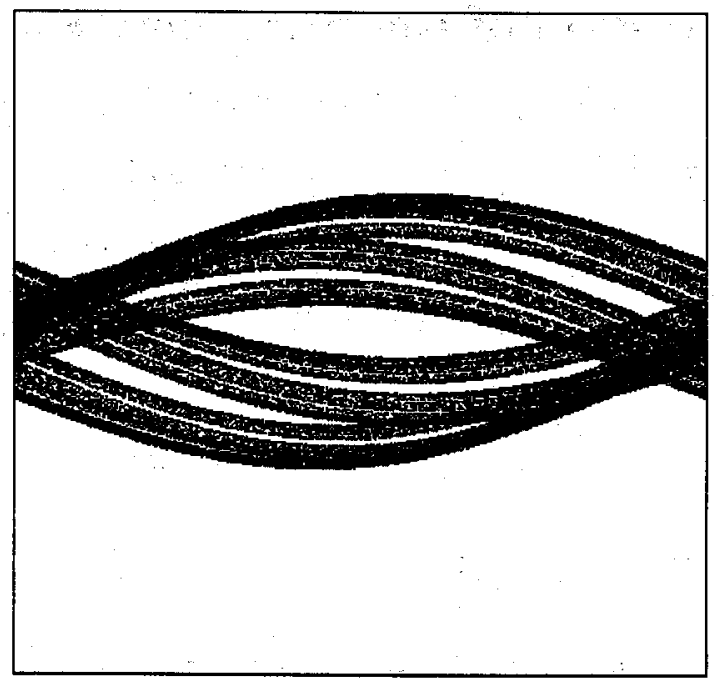

Figure 1: The orbit of the point $(0.1,0)$ up to time 100000 when $\ell=2, \lambda=0.51$ and $f(x)=\sin (x)$.

As simple consequences, we obtain

Corollary $2 \mathcal{D}$ contains an open and dense subset of $(1 / \ell, 1) \times C^{2}\left(S^{1}, \mathbb{R}\right)$.

Corollary 3 For $\ell^{-1}<\lambda<1$ and $2 \leq r \leq \infty$, the set of functions

$$
\mathcal{D}_{\lambda}^{r}=\left\{f \in C^{r}\left(S^{1}, \mathbb{R}\right) \mid(\lambda, f) \in \mathcal{D}^{\circ}\right\}
$$

is an open and dense subset of $C^{r}\left(S^{1}, \mathbb{R}\right)$.

Moreover, the claim of theorem 1 implies that the subset $\mathcal{D}_{\lambda}^{r}$ above occupies almost everywhere in $C^{r}\left(S^{1}, \mathbb{R}\right)$. In fact, if $C^{r}\left(S^{1}, \mathbb{R}\right)$ were a finite dimensional Euclidean space, the claim would imply that the subset $\mathcal{D}_{\lambda}^{r}$ had full measure with respect to the 'Lebesgue measure' on $C^{r}\left(S^{1}, \mathbb{R}\right)$. See [5] and [6] for discussions about measure-theoretical conditions that imply "almost everywhere" for subsets in infinite dimensional spaces.

The proof of theorem 1 is based on an idea that transversality of the unstable manifolds leads to absolute continuity of the SBR measure. We took this idea from a paper of Solomyak and Peres[4] where the authors gave a simplified proof of the above mentioned result of Solomyak.

Acknowledgment: The author like to express his gratitude to Prof. Lai Sang Young for suggesting the papers [1] and [4] in the first stage of this study. 


\section{A description of the SBR measure}

Let $\ell \geq 2, \ell^{-1}<\lambda<1$ and $f \in C^{2}\left(S^{1}, \mathbb{R}\right)$. Consider a map $\tau$ given by

$$
\tau: S^{1} \rightarrow S^{1}, \quad x \mapsto \ell x \quad \bmod \mathbb{Z} .
$$

Let $\mathcal{A}=\{1,2, \cdots, \ell\}$ and let $\mathcal{A}^{p}$ be the space of words of length $p$ on $\mathcal{A}$. For a word $\mathbf{a}=\left(a_{i}\right)_{i=1}^{p} \in \mathcal{A}^{p}$, let us denote its truncation to length $1 \leq q \leq p$ by $[\mathbf{a}]_{q}=\left(a_{i}\right)_{i=1}^{q}$. Let $\mathcal{P}$ be the partition of $S^{1}$ into the intervals

$$
\mathcal{P}(k)=[(k-1) / \ell, k / \ell), \quad k \in \mathcal{A} .
$$

Then $\mathcal{P}^{p}:=\bigvee_{i=0}^{p-1} \tau^{-i}(\mathcal{P})$ is the partition into the intervals

$$
\mathcal{P}(\mathbf{a})=\bigcap_{i=0}^{p-1} \tau^{-i}\left(\mathcal{P}\left(a_{p-i}\right)\right), \quad \mathbf{a}=\left(a_{i}\right)_{i=1}^{p} \in \mathcal{A}^{p} .
$$

For a point $x \in S^{1}$ and $\mathbf{a} \in \mathcal{A}^{p}$, we will denote, by a $(x)$, the unique point $y \in \mathcal{P}(\mathbf{a})$ such that $\tau^{p}(y)=x$. We have $\tau(\mathbf{a}(x))=[\mathbf{a}]_{p-1}(x)$ for $\mathbf{a} \in \mathcal{A}^{p}$.

For a word $\mathbf{a} \in \mathcal{A}^{p}$ of finite length, the image of the segment $\mathcal{P}(\mathbf{a}) \times\{0\} \subset$ $S^{1} \times \mathbb{R}$ under $T^{p}$ is the graph of the function

$$
S(x, \mathbf{a}):=\sum_{i=1}^{p} \lambda^{i-1} f\left(\tau^{p-i}(\mathbf{a}(x))\right)=\sum_{i=1}^{p} \lambda^{i-1} f\left([\mathbf{a}]_{i}(x)\right) .
$$

For a word $\mathbf{a}=\left(a_{i}\right)_{i=1}^{\infty} \in \mathcal{A}^{\infty}$ of infinite length, we define

$$
S(x, \mathbf{a})=\lim _{i \rightarrow \infty} S\left(x,[\mathbf{a}]_{i}\right)=\sum_{i=1}^{\infty} \lambda^{i-1} f\left([\mathbf{a}]_{i}(x)\right) .
$$

Remark that this limit exists and $C^{2}$ as a function of $x$ except for the point 0 , because we have

$$
\sum_{i=1}^{\infty}\left|\lambda^{i-1} \frac{d^{r}}{d x^{r}}\left(f\left([\mathbf{a}]_{i}(x)\right)\right)\right| \leq \sum_{i=1}^{\infty} \lambda^{i-1} \ell^{-i r}\|f\|_{C^{2}}<\infty \quad \text { for } r=0,1,2 .
$$

where

$$
\|f\|_{C^{2}}=\sup _{x \in S^{1}} \max \left\{|f(x)|,\left|\frac{d}{d x} f(x)\right|,\left|\frac{d^{2}}{d x^{2}} f(x)\right|\right\} .
$$

We put $\alpha:=(1-\lambda)^{-1}\|f\|_{C^{2}}$. Then, from the estimate (4), we have

$$
\sup _{x \in S^{1} \backslash\{0\}} \max \left\{|S(x, \mathbf{a})|,\left|\frac{d}{d x} S(x, \mathbf{a})\right|,\left|\frac{d^{2}}{d x^{2}} S(x, \mathbf{a})\right|\right\} \leq \alpha .
$$

for any word $\mathbf{a} \in \mathcal{A}^{p}$ of finite or infinite length $1 \leq p \leq \infty$. Also we have

$$
\left|\frac{d^{r}}{d x^{r}} S(x, \mathbf{a u})-\frac{d^{r}}{d x^{r}} S(x, \mathbf{a})\right| \leq \sum_{i=q+1}^{\infty} \lambda^{i-1} \ell^{-i r}\|f\|_{C^{2}} \leq \lambda^{q} \ell^{-q r} \cdot \alpha
$$


for $\mathbf{a} \in \mathcal{A}^{q}(q<\infty), \mathbf{u} \in \mathcal{A}^{\infty}$ and $r=0,1,2$.

For $x \in S^{1}$, take $\pi(x) \in \mathcal{A}$ so that $\mathcal{P}(\pi(x))$ contains $x$. Then we have

$$
S(\tau(x), \pi(x) \mathbf{a})=f(x)+\lambda S(x, \mathbf{a}) .
$$

for any word $\mathbf{a} \in \mathcal{A}^{p}$ of length $1 \leq p \leq \infty$. Let us consider maps

$$
\Psi: S^{1} \times \mathcal{A}^{\infty} \rightarrow S^{1} \times \mathbb{R}, \quad \Psi(x, \mathbf{a})=(x, S(x, \mathbf{a}))
$$

and

$$
\Theta: S^{1} \times \mathcal{A}^{\infty} \rightarrow S^{1} \times \mathcal{A}^{\infty}, \quad \Theta(x, \mathbf{a})=(\tau(x), \pi(x) \mathbf{a}) .
$$

Then (7) gives a commutative relation

$$
T \circ \Psi=\Psi \circ \Theta: S^{1} \times \mathcal{A}^{\infty} \rightarrow S^{1} \times \mathbb{R} .
$$

Let $\mathrm{m}$ be the normalized Lebesgue measure on $S^{1}$ and let $\nu$ be the Bernoulli measure on $\mathcal{A}^{\infty}$, that is, the infinite product of the probability measure on $\mathcal{A}$ that gives equal probability $1 / \ell$ for each element. The product $\mathbf{m} \times \nu$ is invariant and ergodic with respect to the map $\Theta$. Hence the measure $\mu=\Psi(\mathbf{m} \times \nu)$ is invariant and ergodic with respect to $T$ from the commutative relation above. Since the map $T$ is contracting in the vertical direction and since the projection of $\mu$ to $S^{1}$ equals to $\mathrm{m}$, Lebesgue almost every point in $S^{1} \times \mathbb{R}$ is generic for the measure $\mu$, that is, $\mu$ is the SBR measure for $T$.

\section{A bilinear form of the space of measures}

For a positive real number $r$, we define a non-negative symmetric bilinear form $(\cdot, \cdot)_{r}$ on the space of finite signed Borel measures on $\mathbb{R}$ by

$$
\left(\rho, \rho^{\prime}\right)_{r}=\int_{\mathbb{R}} \rho(B(z, r)) \rho^{\prime}(B(z, r)) d z
$$

where $B(z, r)=\{y \in \mathbb{R}|| z-y \mid<r\}$. Put $\|\rho\|_{r}=\sqrt{(\rho, \rho)_{r}}$. As a version of Schwarz inequality, we have

$$
\left(\rho, \rho^{\prime}\right)_{r} \leq\|\rho\|_{r} \cdot\left\|\rho^{\prime}\right\|_{r}
$$

Lemma 4 If liminf $\lim _{r \rightarrow+0} r^{-1}\|\rho\|_{r}<\infty$ for a Borel probability measure $\rho$ on $\mathbb{R}$, then $\rho$ is absolutely continuous with respect to the Lebesgue measure on $\mathbb{R}$ and the density function $d \mu / d x$ satisfies $\|d \mu / d x\|_{L^{2}} \leq \liminf _{r \rightarrow+0} r^{-1}\|\rho\|_{r}$.

Proof. For $r>0$, let us define $g_{r}(x)=r^{-1} \rho(B(x, r))$. Then $\left\|g_{r}\right\|_{L^{2}}=r^{-1}\|\rho\|_{r}$. The assumption implies that there exists a sequence $r(k) \rightarrow 0$ such that

$$
\lim _{k \rightarrow \infty}\left\|g_{r(k)}\right\|_{L^{2}} \leq \liminf _{r \rightarrow+0} r^{-1}\|\rho\|_{r}
$$


Hence, taking a subsequence if necessary, we can assume that the sequence $g_{r(k)}$ converges weakly to some $g_{\infty} \in L^{2}(\mathbb{R})$ such that $\left\|g_{\infty}\right\|_{L^{2}} \leq \liminf _{r \rightarrow+0} r^{-1}\|\rho\|_{r}$ The weak convergence implies

$$
\int_{\mathbb{R}} h g_{\infty} d x=\lim _{k \rightarrow \infty} \int_{\mathbb{R}} h g_{r(k)} d x=\int_{\mathbb{R}} h d \mu
$$

for every continuous function $h$ with compact support. Hence $g_{\infty}=d \mu / d x$.

Let $\mu_{x}=\Psi\left(\delta_{x} \times \nu\right)$ for $x \in S^{1}$, where $\delta_{x}$ is the point measure at $x$. This family of measures $\mu_{x}, x \in S^{1}$, is the conditional measures of the SBR measure $\mu$ with respect to the partition of $S^{1} \times \mathbb{R}$ into the fibers $\{x\} \times \mathbb{R}, x \in S^{1}$. Put

$$
I(r)=r^{-2} \int_{S^{1}}\left\|\mu_{x}\right\|_{r}^{2} d x
$$

As a corollary of the last lemma, we can give the following sufficient condition for the SBR measure $\mu$ to be absolutely continuous.

- Corollary 5 If $\liminf _{r \rightarrow 0} I(r)<\infty$, the $S B R$ measure $\mu$ is absolutely continuous with respect to the Lebesgue measure on $S^{1} \times \mathbb{R}$ and the density function is square integrable.

Proof. From Fatou's inequality, we have

$$
\int_{S^{1}} \liminf _{r \rightarrow 0} r^{-2}\left\|\mu_{x}\right\|_{r}^{2} d x \leq \liminf _{r \rightarrow+0} I(r)<\infty
$$

This and the last lemma give the claim.

\section{Transversality}

In this section, we show that the SBR measure for $T$ is absolutely continuous if most pairs of the graphs of $S(x, \mathbf{a}), \mathbf{a} \in \mathcal{A}^{\infty}$, are transversal to each other. Actually, if we assumed that all pairs were transversal to each other, the argument below would be simpler. But we do not know whether such assumption is a generic one. (The author suspects it is not.) Here we will give a weaker alternative sufficient condition for the SBR measure to be absolutely continuous.

The invariance of the SBR measure $\mu$ w.r.t. $T$ gives the relation

$$
\mu_{\boldsymbol{x}}=\ell^{-q} \sum_{\mathbf{a} \in \mathcal{A}^{q}} T^{q}\left(\mu_{\mathbf{a}(x)}\right)
$$

for $q \geq 1$ and $x \in S^{1}$. For $\mathrm{c} \in \mathcal{A}^{p}$ and $\mathrm{a}, \mathrm{b} \in \mathcal{A}^{q}$, we put

$$
I(r, \mathbf{c})=r^{-2} \int_{\mathcal{P}(\mathbf{c})}\left\|\mu_{x}\right\|_{r}^{2} d \mathbf{m}(x)
$$

and

$$
I(r, \mathbf{c} ; \mathbf{a}, \mathbf{b})=r^{-2} \int_{\mathcal{P}(\mathbf{c})}\left(T^{q}\left(\mu_{\mathbf{a}(x)}\right), T^{q}\left(\mu_{\mathbf{b}(x)}\right)\right)_{r} d \mathbf{m}(x)
$$


From (9), we have

$$
I(r, \mathbf{c})=\ell^{-2 q} \sum_{\mathbf{a}, \mathbf{b} \in \mathcal{A}^{q}} I(r, \mathbf{c} ; \mathbf{a}, \mathbf{b}) .
$$

The function $S(\cdot$, a) for a word a may not be continuous on the closure of $\mathcal{P}(\mathbf{c})$ if $\mathcal{P}(\mathbf{c})$ has 0 as its right endpoint. But, obviously, the restriction of $S(\cdot$, a) to $\mathcal{P}(\mathbf{c})$ can be extended to the closure of $\mathcal{P}(\mathbf{c})$ as a $C^{2}$ function. We will denote this extension by $S_{\mathrm{c}}(\cdot, \mathrm{a})$.

Let us consider words $\mathbf{a}, \mathbf{b} \in \mathcal{A}^{q}$ and $\mathbf{c} \in \mathcal{A}^{p}$. We say that the functions $S(\cdot, \mathbf{a})$ and $S(\cdot, \mathbf{b})$ are $(\epsilon, \delta)$-transversal on $\mathcal{P}(\mathbf{c})$ if either

$$
\left|S_{\mathrm{c}}(x, \mathbf{a})-S_{\mathrm{c}}(x, \mathbf{b})\right|>\epsilon \quad \text { or } \quad\left|\frac{d}{d x} S_{\mathrm{c}}(x, \mathbf{a})-\frac{d}{d x} S_{\mathrm{c}}(x, \mathbf{b})\right|>\delta
$$

holds for each point $x$ in the closure of $\mathcal{P}(\mathbf{c})$. Otherwise we will say that they are $(\epsilon, \delta)$-tangent on $\mathcal{P}(c)$.

Proposition 6 Let $\mathbf{a}, \mathbf{b} \in \mathcal{A}^{q}$ and $\mathbf{c} \in \mathcal{A}^{p}$. If the functions $S(\cdot$, au) and $S(\cdot, \mathrm{bv})$ are $(\epsilon, \delta)$-transversal on $\mathcal{P}(\mathbf{c})$ for every $\mathbf{u}$ and $\mathbf{v}$ in $\mathcal{A}^{\infty}$, then we have

$$
I(r, \mathbf{c} ; \mathbf{a}, \mathbf{b}) \leq 8 \delta^{-1} \ell^{-p} \max \left\{4 \alpha / \epsilon, \ell^{p}\right\}
$$

for $0<r<\epsilon / 4$.

Proof. Let $\chi_{r}(\cdot)$ be the characteristic function of the interval $(-r, r)$. By Fubini's theorem, we have

$$
\begin{aligned}
\left(T^{q}\left(\mu_{\mathbf{a}(x)}\right),\right. & \left.T^{q}\left(\mu_{\mathbf{b}(x)}\right)\right)_{r} \\
& =\int_{\mathbb{R}^{2}}\left(\int_{\mathbb{R}} \chi_{r}(s-z) \chi_{r}(t-z) d z\right) d\left(T^{q}\left(\mu_{\mathbf{a}(x)}\right) \times T^{q}\left(\mu_{\mathbf{b}(x)}\right)\right)(s, t) \\
& \leq 2 r \int_{\mathbb{R}^{2}} \chi_{2 r}(s-t) d\left(T^{q}\left(\mu_{\mathbf{a}(x)}\right) \times T^{q}\left(\mu_{\mathbf{b}(x)}\right)\right)(s, t) \\
& \leq 2 r \int_{\mathcal{A}^{\infty} \times \mathcal{A}^{\infty}} \chi_{2 r}(S(x, \mathbf{a u})-S(x, \mathbf{b v})) d(\nu \times \nu)(\mathbf{u}, \mathbf{v}) .
\end{aligned}
$$

Again by Fubini's theorem, we obtain

$$
\begin{aligned}
& I(r, \mathbf{c} ; \mathbf{a}, \mathbf{b}) \\
& \leq 2 r^{-1} \int_{\mathcal{A}^{\infty} \times \mathcal{A}^{\infty}} \mathbf{m}(\{x \in \mathcal{P}(\mathbf{c})|| S(x, \mathbf{a u})-S(x, \mathbf{b v}) \mid<2 r\}) d(\nu \times \nu)(\mathbf{u}, \mathbf{v}) .
\end{aligned}
$$

Hence the claim of the proposition follows if we show

$$
\mathbf{m}(\{x \in \mathcal{P}(\mathbf{c})|| S(x, \mathbf{a u})-S(x, \text { bv })) \mid<2 r\}) \leq 4 r \delta^{-1} \ell^{-p} \max \left\{4 \alpha / \epsilon, \ell^{p}\right\}
$$

for every $\mathbf{u}$ and $\mathbf{v}$ in $\mathcal{A}^{\infty}$. Take an arbitrary connected component $J$ of the set

$$
\{x \in \mathcal{P}(\mathrm{c})|| S(x, \mathrm{au})-S(x, \mathrm{bv}) \mid<\epsilon\}
$$


and put

$$
B=\{x \in J|| S(x, \mathbf{a u})-S(x, \mathbf{b v}) \mid<2 r\} \subset J .
$$

In order to show the inequality (12), it is enough to prove

$$
\mathrm{m}(B) / \mathrm{m}(J) \leq 4 r \delta^{-1} \max \left\{4 \alpha / \epsilon, \ell^{p}\right\} .
$$

If $B=\emptyset,(13)$ is trivial. Assume $B \neq \emptyset$. From (5) and the $(\epsilon, \delta)$-transversality of $S(\cdot, \mathbf{a u})$ and $S(\cdot, \mathrm{bv})$, we have, for $x \in J$,

$$
\delta<\left|\frac{d}{d x} S(x, \mathbf{a u})-\frac{d}{d x} S(x, \mathbf{b v})\right| \leq 2 \alpha .
$$

Hence $B$ consists of a single interval with length not larger than $4 r / \delta$. On the other hand, the length of one of the connected components of $J \backslash B$ is not less than $(\epsilon-2 r) /(2 \alpha) \geq \epsilon /(4 \alpha)$ unless the component contains an end point of $\mathcal{P}(\mathrm{c})$ in its closure. Thus the length of $J$ is larger than $\epsilon /(4 \alpha)$ or, otherwise, $J=\mathcal{P}(\mathbf{c})$. From these, we obtain

$$
\frac{\mathrm{m}(B)}{\mathrm{m}(J)} \leq \frac{4 r / \delta}{\min \left\{\epsilon /(4 \alpha), \ell^{-p}\right\}}=4 r \delta^{-1} \max \left\{4 \alpha / \epsilon, \ell^{p}\right\}
$$

We have completed the proof of proposition 6 .

For $1 \leq p, q<\infty, \mathbf{c} \in \mathcal{A}^{q}$ and $\epsilon, \delta>0$, let $\mathbf{E}(q, \mathbf{c} ; \epsilon, \delta)$ be the set of pairs $(\mathrm{a}, \mathrm{b}) \in \mathcal{A}^{q} \times \mathcal{A}^{q}$ for which there exist $\mathrm{u}$ and $\mathbf{v}$ in $\mathcal{A}^{\infty}$ such that the functions $S(\cdot, \mathbf{a u})$ and $S(\cdot, \mathbf{b v})$ are $(\epsilon, \delta)$-tangent on $\mathcal{P}(\mathbf{c})$. We define

$$
\mathbf{e}(q, p ; \epsilon, \delta)=\max _{\mathbf{c} \in \mathcal{A}^{p}} \max _{\mathbf{a} \in \mathcal{A}^{q}} \#\left\{\mathbf{b} \in \mathcal{A}^{q} \mid(\mathbf{a}, \mathbf{b}) \in \mathbf{E}(q, \mathbf{c} ; \epsilon, \delta)\right\}
$$

where $\#\{\cdot\}$ is the cardinality of the set $\{\cdot\}$. Obviously $\mathbf{e}(q, p ; \epsilon, \delta)$ is decreasing with respect to $p$ and increasing with respect to $\epsilon$ and $\delta$. We put

$$
\mathbf{e}(q)=\min \{\mathbf{e}(q, p ; \epsilon, \delta) \mid \epsilon>0, \delta>0, p \geq 1\}=\lim _{p \rightarrow \infty} \lim _{\epsilon \rightarrow+0} \lim _{\delta \rightarrow+0} \mathbf{e}(q, p ; \epsilon, \delta) .
$$

Proposition 7 We have

$$
I(r) \leq \lambda^{-q} \ell^{-q} \mathbf{e}(q, p ; \epsilon, \delta) I\left(\lambda^{-q} r\right)+8 \delta^{-1} \max \left\{4 \alpha / \epsilon, \ell^{p}\right\}
$$

for $1 \leq p, q<\infty, \epsilon>0, \delta>0$ and $0<r<\epsilon / 4$.

Proof. Notice that, from (11),

$$
I(r)=\sum_{\mathbf{c} \in \mathcal{A}^{p}} \sum_{(\mathbf{a}, \mathbf{b}) \in \mathcal{A}^{q} \times \mathcal{A}^{q}} \ell^{-2 q} \cdot I(r, \mathbf{c} ; \mathbf{a}, \mathbf{b}) .
$$

From proposition 6 , we have

$$
\sum_{\mathbf{c} \in \mathcal{A}^{p}} \sum_{(\mathbf{a}, \mathbf{b}) \notin \mathbf{E}(q, \mathbf{c} ; \epsilon, \delta)} \ell^{-2 q} I(r, \mathbf{c} ; \mathbf{a}, \mathbf{b}) \leq 8 \delta^{-1} \max \left\{4 \alpha / \epsilon, \ell^{p}\right\}
$$


Since $T^{q}$ is a contraction of rate $\lambda^{q}$ in vertical direction, we have

$$
\left(T^{q}\left(\mu_{\mathbf{a}(x)}\right), T^{q}\left(\mu_{\mathbf{a}(x)}\right)\right)_{r}=\lambda^{q}\left(\mu_{\mathbf{a}(x)}, \mu_{\mathbf{a}(x)}\right)_{\lambda^{-q_{r}}} \quad \text { for } \mathbf{a} \in \mathcal{A}^{q} .
$$

From the Schwarz inequality (8) and the equality (17), we have

$$
\begin{aligned}
\sum_{(\mathbf{a}, \mathbf{b}) \in \mathbf{E}(q, \mathbf{c} ; \epsilon, \delta)}\left(T^{q}\left(\mu_{\mathbf{a}(x)}\right), T^{q}\left(\mu_{\mathbf{b}(x)}\right)\right)_{r} & \leq \sum_{(\mathbf{a}, \mathbf{b}) \in \mathbf{E}(q, \mathbf{c} ; \epsilon, \delta)}\left\|T^{q}\left(\mu_{\mathbf{a}(x)}\right)\right\|_{r} \cdot\left\|T^{q}\left(\mu_{\mathbf{b}(x)}\right)\right\|_{r} \\
& \leq \lambda^{q} \sum_{(\mathbf{a}, \mathbf{b}) \in \mathbf{E}(q, \mathbf{c} ; \epsilon, \delta)}\left\|\mu_{\mathbf{a}(x)}\right\|_{\lambda^{-q_{r}}} \cdot\left\|\mu_{\mathbf{b}(x)}\right\|_{\lambda^{-q_{r}}} \\
& \leq \lambda^{q} \sum_{(\mathbf{a}, \mathbf{b}) \in \mathbf{E}(q, \mathbf{c} ; \epsilon, \delta)} \frac{\left\|\mu_{\mathbf{a}(x)}\right\|_{\lambda^{-q_{r}}}^{2}+\left\|\mu_{\mathbf{b}(x)}\right\|_{\lambda^{-q_{r}}}^{2}}{2} \\
& \leq \lambda^{q} \cdot \mathbf{e}(q, p ; \epsilon, \delta) \sum_{\mathbf{a} \in \mathcal{A}^{q}}\left\|\mu_{\mathbf{a}(x)}\right\|_{\lambda^{-q_{r}}}^{2} .
\end{aligned}
$$

Multiplying both sides by $r^{-2} \ell^{-2 q}$ and integrating them over $S^{1}$, we obtain

$$
\begin{aligned}
\sum_{\mathbf{c} \in \mathcal{A}^{p}} \sum_{(\mathbf{a}, \mathbf{b}) \in \mathbf{E}(q, \mathbf{c} ; \epsilon, \delta)} & \ell^{-2 q} I(r, \mathbf{c} ; \mathbf{a}, \mathbf{b}) \\
& \leq r^{-2} \ell^{-2 q} \lambda^{q} \cdot \mathbf{e}(q, p ; \epsilon, \delta) \sum_{\mathbf{a} \in \mathcal{A}^{q}}\left(\int_{S^{1}}\left\|\mu_{\mathbf{a}(x)}\right\|_{\lambda^{-q} r}^{2} d x\right) \\
& \leq r^{-2} \ell^{-2 q} \lambda^{q} \cdot \mathbf{e}(q, p ; \epsilon, \delta) \sum_{\mathbf{a} \in \mathcal{A}^{q}}\left(\ell^{q} \int_{\mathcal{P}(\mathbf{a})}\left\|\mu_{y}\right\|_{\lambda^{-q} r}^{2} d y\right) \\
& \leq \lambda^{-q} \ell^{-q} \cdot \mathbf{e}(q, p ; \epsilon, \delta) I\left(\lambda^{-q} r\right) .
\end{aligned}
$$

The inequality (14) follows from (15), (16) and the last inequality.

Proposition 8 If $\mathbf{e}(q)<\lambda^{q} \ell^{q}$ for some positive integer $q$, the combination $(\lambda, f)$ is contained in $\mathcal{D}^{\circ}$.

Proof. Choose large $p$ and small $\epsilon>0, \delta>0$ so that $\mathbf{e}(q, p ; \epsilon, \delta)=\mathbf{e}(q)$. From proposition 7, we have, for $r<\epsilon / 4$,

$$
I(r) \leq \beta \cdot I\left(\lambda^{-q} r\right)+R
$$

where $\beta=\lambda^{-q} \ell^{-q} \mathbf{e}(q)$ and $R=8 \delta^{-1} \max \left\{4 \alpha / \epsilon, \ell^{p}\right\}$. It follows inductively that

$$
I\left(\lambda^{n q} \epsilon / 4\right) \leq \beta^{n} I(\epsilon / 4)+\sum_{i=0}^{n-1} \beta^{i} R \quad \text { for } \quad n=1,2,3, \cdots
$$

Since $\beta<1$ from the assumption, we obtain

$$
\liminf _{r \rightarrow 0} I(r) \leq(1-\beta)^{-1} R .
$$

From corollary 5 , the SBR measure for $T$ is absolutely continuous. 
Remark that the functions $S(\cdot, \mathbf{a})$ depend continuously on $\mathbf{a} \in \mathcal{A}^{\infty}, \lambda \in \mathbb{R}$ and $f \in C^{2}\left(S^{1}, \mathbb{R}\right)$ in $C^{2}$ sense. (See the definition (3) and the estimate (4).) Thus $(\epsilon, \delta)$-transversality of a pair of those functions is an open condition on $\mathrm{a} \in \mathcal{A}^{\infty}, \lambda$ and $f$. By a standard argument using compactness, we see that $\mathbf{e}(q)$ is upper semicontinuous with respect to $f$. Therefore $f$ is contained in $\mathcal{D}^{\circ}$.

Proposition 8 implies that a pair $(\lambda, f)$ belongs to $\mathcal{D}^{\circ}$ if the growth rate of the quantity $\mathrm{e}(q)$ as $q$ increase is smaller than $\lambda \ell$. Below we estimate this growth rate using another quantity.

For $q \geq 1$ and $\mathbf{c} \in \mathcal{A}^{p}$, let $\mathbf{d}(q, \mathbf{c} ; \epsilon, \delta)$ be the maximum cardinality of a subset $\left\{\mathbf{a}_{1}, \mathbf{a}_{2}, \cdots, \mathbf{a}_{d}\right\} \subset \mathcal{A}^{q}$ satisfying the conditions

(a) the first alphabets $\left[\mathbf{a}_{i}\right]_{1} \in \mathcal{A}, 1 \leq i \leq d$, are mutually distinct and

(b) $S\left(\cdot, \mathbf{a}_{i}\right)$ and $S\left(\cdot, \mathbf{a}_{j}\right)$ are $\left(4 \lambda^{q} \alpha+\epsilon, 4 \lambda^{q} \ell^{-q} \alpha+\delta\right)$-tangent on $P(\mathbf{c})$.

Obviously we have $\mathbf{d}(q, \mathbf{c} ; \epsilon, \delta) \leq \ell$ from the first condition (a). Let us put $\mathrm{d}(q, p ; \epsilon, \delta)=\max _{\mathbf{c} \in \mathcal{A}^{p}} \mathrm{~d}(q, \mathbf{c} ; \epsilon, \delta)$. Then $\mathbf{d}(q, p ; \epsilon, \delta)$ is decreasing with respect to $p$ and increasing with respect to $\epsilon, \delta>0$. We put

$$
\mathbf{d}(q)=\min \{\mathbf{d}(q, p ; \epsilon, \delta) \mid \epsilon>0, \delta>0, p \geq 1\} .
$$

Proposition $9 \quad \mathrm{e}(q) \leq \prod_{i=1}^{q} \mathrm{~d}(i)$.

Proof. Take $\epsilon, \delta>0$ so small and $p$ so large that

$$
\mathbf{d}(q-i)=\mathbf{d}\left(q-i, p+i ; 2 \lambda^{-i}\left(\ell^{-p} \alpha+\epsilon\right), 2 \lambda^{-i} \ell^{i}\left(\ell^{-p} \alpha+\delta\right)\right)
$$

for all $0 \leq i \leq q-1$. Let us consider $\mathrm{c} \in \mathcal{A}^{p}$ and $\mathrm{a} \in \mathcal{A}^{q}$. Notice that, if a pair $(\mathbf{a}, \mathbf{b}) \in \mathcal{A}^{q} \times \mathcal{A}^{q}$ is contained in the set $E(q, \mathbf{c} ; \epsilon, \delta)$, the functions $S(\cdot, \mathbf{a})$ and $S(\cdot, \mathbf{b})$ are $\left(2 \lambda^{q} \alpha+\epsilon, 2 \lambda^{q} \ell^{-q} \alpha+\delta\right)$-tangent on $\mathcal{P}(\mathbf{c})$ from the estimate $(6)$. Let $W(\mathbf{a}, \mathbf{c})$ be the set of elements $\mathbf{b} \in \mathcal{A}^{q}$ such that the functions $S(\cdot, \mathbf{a})$ and $S(\cdot, \mathbf{b})$ are $\left(2 \lambda^{q} \alpha+\epsilon, 2 \lambda^{q} \ell^{-q} \alpha+\delta\right)$-tangent on $\mathcal{P}(\mathbf{c})$. Then

$$
\mathbf{e}(q) \leq \mathbf{e}(q, p ; \epsilon, \delta) \leq \max _{\mathbf{c} \in \mathcal{A}^{p}} \max _{\mathbf{a} \in \mathcal{A}^{q}} \# W(\mathbf{a}, \mathbf{c}) .
$$

For the proof of proposition 9 , it is enough to show

$$
\# W(\mathbf{a}, \mathbf{c}) \leq \prod_{i=0}^{q-1} \mathbf{d}\left(q-i, p+i ; 2 \lambda^{-i}\left(\ell^{-p} \alpha+\epsilon\right), 2 \lambda^{-i} \ell^{i}\left(\ell^{-p} \alpha+\delta\right)\right)
$$

for arbitrary $\mathrm{c} \in \mathcal{A}^{p}$ and $\mathbf{a} \in \mathcal{A}^{q}$. We prepare a lemma for the proof of (18).

Lemma 10 For any $\mathbf{b}_{1}$ and $\mathbf{b}_{2}$ in $W(\mathbf{a}, \mathbf{c})$, the functions $S\left(\cdot, \mathbf{b}_{1}\right)$ and $S\left(\cdot, \mathbf{b}_{2}\right)$ are $\left(4 \lambda^{q} \alpha+2 \epsilon+2 \ell^{-p} \alpha, 4 \lambda^{q} \ell^{-q} \alpha+2 \delta+2 \ell^{-p} \alpha\right)$-tangent on $\mathcal{P}(\mathbf{c})$.

Proof. From the definition of $W(\mathbf{a}, \mathbf{c})$, there exist points $x_{i}, i=1,2$, in the closure of $\mathcal{P}(\mathbf{c})$ such that $\left|S_{\mathbf{c}}\left(x_{i}, \mathbf{b}_{i}\right)-S_{\mathbf{c}}\left(x_{i}, \mathbf{a}\right)\right| \leq 2 \lambda^{q} \alpha+\epsilon$ and

$$
\left|\frac{d S_{\mathbf{c}}}{d x}\left(x_{i}, \mathbf{b}_{i}\right)-\frac{d S_{\mathrm{c}}}{d x}\left(x_{i}, \mathbf{a}\right)\right| \leq 2 \lambda^{q} \ell^{-q} \alpha+\delta .
$$


From (5), the variations of $S_{\mathbf{c}}(\cdot, \mathbf{w})$ and $\frac{d}{d x} S_{\mathbf{c}}(\cdot, \mathbf{w})$ on $\mathcal{P}(\mathbf{c})$ is bounded by $\ell^{-p} \alpha$ for any word $\mathrm{w} \in \mathcal{A}^{q}$. Thus we have.

$$
\begin{aligned}
\left|S_{\mathbf{c}}\left(x_{1}, \mathbf{b}_{1}\right)-S_{\mathbf{c}}\left(x_{1}, \mathbf{b}_{2}\right)\right| \leq & \left|S_{\mathbf{c}}\left(x_{1}, \mathbf{b}_{1}\right)-S_{\mathbf{c}}\left(x_{1}, \mathbf{a}\right)\right|+\left|S_{\mathbf{c}}\left(x_{1}, \mathbf{a}\right)-S_{\mathbf{c}}\left(x_{2}, \mathbf{a}\right)\right| \\
& +\left|S_{\mathbf{c}}\left(x_{2}, \mathbf{a}\right)-S_{\mathbf{c}}\left(x_{2}, \mathbf{b}_{2}\right)\right|+\left|S_{\mathbf{c}}\left(x_{2}, \mathbf{b}_{2}\right)-S_{\mathbf{c}}\left(x_{1}, \mathbf{b}_{2}\right)\right| \\
\leq & 4 \lambda^{q} \alpha+2 \epsilon+2 \ell^{-p} \alpha
\end{aligned}
$$

and, similarly,

$$
\left|\frac{d S_{\mathbf{c}}}{d x}\left(x_{1}, \mathbf{b}_{1}\right)-\frac{d S_{\mathbf{c}}}{d x}\left(x_{1}, \mathbf{b}_{2}\right)\right| \leq 4 \lambda^{q} \ell^{-q} \alpha+2 \delta+2 \ell^{-p} \alpha .
$$

These imply the claim of lemma 10.

Let $W_{i}=\left\{[\mathbf{b}]_{i} \mid \mathbf{b} \in W(\mathbf{a}, \mathbf{c})\right\} \subset \mathcal{A}^{i}$, for $1 \leq i \leq q$. Obviously $W_{q}=W(\mathbf{a}, \mathbf{c})$. From lemma $10, \# W_{1} \leq \mathbf{d}\left(q, p ; 2\left(\ell^{-p} \alpha+\epsilon\right), 2\left(\ell^{-p} \alpha+\delta\right)\right)$. Hence, in order to prove (18), it is enough show

$$
\# W_{i+1} \leq \mathrm{d}\left(q-i, p+i ; 2 \lambda^{-i}\left(\ell^{-p} \alpha+\epsilon\right), 2 \lambda^{-i} \ell^{i}\left(\ell^{-p} \alpha+\delta\right)\right) \cdot \# W_{i}
$$

for $1 \leq i \leq q-1$. Take an arbitrary element $\mathbf{b} \in W_{i}$ and let $\mathbf{b}_{1}, \mathbf{b}_{2}, \cdots, \mathbf{b}_{m}$ be the elements of $W(\mathbf{a}, \mathbf{c})$ such that $\left[\mathbf{b}_{j}\right]_{i}=\mathbf{b}$ and that $\left[\mathbf{b}_{j}\right]_{i+1}$ are mutually distinct. Then we can write each $\mathbf{b}_{j}$ as $\mathbf{b}_{j}=\mathbf{b} \mathbf{u}_{j}$ by using a word $\mathbf{u}_{j} \in \mathcal{A}^{q-i}$. From lemma 10 , the functions $S\left(\cdot, \mathbf{b}_{j}\right)$ are $\left(4 \lambda^{q} \alpha+2 \epsilon+2 \ell^{-p} \alpha, 4 \lambda^{q} \ell^{-q} \alpha+2 \delta+2 \ell^{-p} \alpha\right)-$ tangent to each other on $\mathcal{P}(\mathrm{c})$. Since we have

$$
S\left(x, \mathbf{b}_{j}\right)=S(x, \mathbf{b})+\lambda^{i} S\left(\mathbf{b}(x), \mathbf{u}_{j}\right)
$$

from the definition (2), this implies that the functions $S\left(\cdot, \mathbf{u}_{j}\right), 1 \leq j \leq m$, are

$$
\left(4 \lambda^{q-i} \alpha+\lambda^{-i}\left(2 \epsilon+2 \ell^{-p} \alpha\right), 4 \lambda^{q-i} \ell^{-(q-i)} \alpha+\lambda^{-i} \ell^{i}\left(2 \delta+2 \ell^{-p} \alpha\right)\right)
$$

-tangent to each other on $\mathcal{P}(\mathbf{b c})$. Remark that the first alphabet of $\mathbf{u}_{j}$ 's are mutually distinct. Thus we have

$$
m \leq \mathbf{d}\left(q-i, p+i ; 2 \lambda^{-i}\left(\ell^{-p} \alpha+\epsilon\right), 2 \lambda^{-i} \ell^{i}\left(\ell^{-p} \alpha+\delta\right)\right) .
$$

Since this holds for arbitrary $\mathbf{b} \in W_{i}$, we obtain (19).

From proposition 8 and proposition 9 , we get another sufficient condition, which we will make use of in section 6 .

Corollary 11 A combination $(\lambda, f) \in \mathbb{R} \times C^{2}\left(S^{1}, \mathbb{R}\right)$ is contained in $\mathcal{D}^{\circ}$ if

$$
\limsup _{q \rightarrow \infty} \mathrm{d}(q)<\lambda \ell \text {. }
$$

\section{Generic families}

In this section and the next, we will consider families of maps of the form (1). Henceforth we fix an integer $\ell \geq 2$ and a real number $\lambda$ such that $\ell^{-1}<\lambda<1$. 
Let $g$ be a $C^{2}$ function on $S^{1}$ and let $\varphi_{i}, 1 \leq i \leq m$, be $C^{\infty}$ functions on $S^{1}$. For these functions, we consider a family of functions

$$
f_{\mathfrak{t}}(x)=g(x)+\sum_{i=1}^{m} t_{i} \varphi_{i}(x): S^{1} \rightarrow \mathbb{R}
$$

with parameters $\mathbf{t}=\left(t_{1}, t_{2}, \cdots, t_{m}\right) \in \mathbb{R}^{m}$. Then the corresponding family of maps is

$$
T_{\mathrm{t}}: S^{1} \times \mathbb{R} \rightarrow S^{1} \times \mathbb{R}, \quad T_{\mathbf{t}}(x, y)=\left(\ell x, \lambda y+f_{\mathrm{t}}(x)\right) .
$$

Remark that $\alpha, \mathrm{d}(q, p ; \epsilon, \delta)$ and $\mathrm{d}(q)$ in the previous sections now depend on the parameter $\mathbf{t}$. We will denote $\alpha(\mathbf{t}), \mathbf{d}(q, p ; \epsilon, \delta ; \mathbf{t})$ and $\mathbf{d}(q ; \mathbf{t})$ for them in order to express the dependence.

In this section, we will show that the limit lim $\sup _{q \rightarrow \infty} \mathbf{d}(q ; \mathbf{t})$ is bounded by a relatively small constant for Lebesgue almost all parameters $t$ under an assumption on the family $T_{\mathrm{t}}$. Actually, the bound is not so good as required in corollary 11 . However, in the next section, we will apply this estimate to the family of the iterates $T_{\mathbf{t}}^{n}$ and prove theorem 1 .

First, we define a Jacobian for linear maps on Euclidean spaces. For an Euclidean space $X$, we denote the Lebesgue measure on it by $\mathbf{L e b}_{X}$ and put

$$
\operatorname{Ball}(\eta ; X)=\{z \in X \mid\|z\| \leq \eta\} .
$$

Let $L: E \rightarrow F$ be a linear map between Euclidean spaces $E$ and $F$. If $L$ is surjective, we define its Jacobian by

$$
\begin{aligned}
\operatorname{Jac}(L) & =\operatorname{Leb}_{F}(\operatorname{Ball}(1 ; F)) / \operatorname{Leb}_{\operatorname{Ker}(L)^{\perp}}\left(L^{-1}(\operatorname{Ball}(1 ; F)) \cap \operatorname{Ker}(L)^{\perp}\right) \\
& =\sup _{H}\left(\operatorname{Leb}_{F}(\operatorname{Ball}(1 ; F)) / \operatorname{Leb}_{H}\left(L^{-1}(\operatorname{Ball}(1 ; F)) \cap H\right)\right)
\end{aligned}
$$

where $\operatorname{Ker}(L)^{\perp}$ is the orthogonal complement of the kernel of $L$ and the supermum on the second line (22) is taken over subspaces $H \subset E$ such that $\operatorname{dim} H=\operatorname{dim} F$. If $L$ is not surjective, we simply put $\operatorname{Jac}(L)=0$. The following are easy consequences of this definition.

Lemma 12 If $L: E \rightarrow F$ is surjective, we have, for any Borel set $X \subset F$,

$$
\operatorname{Leb}_{E}\left(L^{-1}(X) \cap \operatorname{Ball}(\eta ; E)\right) \leq \frac{\operatorname{Leb}_{F}(X) \cdot \operatorname{Leb}_{\operatorname{Ker}(L)}(\operatorname{Ball}(\eta ; \operatorname{Ker}(L)))}{\operatorname{Jac}(L)} .
$$

Lemma 13 Let $E_{0}$ be a subspace of an Euclidean space $E$ and let $L: E \rightarrow F$ be a linear map. For the restriction $\left.L\right|_{E_{0}}$ of $L$ to $E_{0}$, we have $\operatorname{Jac}(L) \geq \operatorname{Jac}\left(\left.L\right|_{E_{0}}\right)$.

For an affine map $A: E \rightarrow F$, we define its Jacobian $\operatorname{Jac}(A)$ as that of its linear part.

Next we state a condition that we call $(\gamma, \delta)$-genericity. Modifying the definition (2) for the family $T_{\mathbf{t}}$, we put

$$
S(x, \mathbf{a} ; \mathbf{t})=\sum_{i=1}^{p} \lambda^{i-1} f_{\mathbf{t}}\left([\mathbf{a}]_{i}(x)\right)
$$


for $\mathbf{t} \in \mathbb{R}^{m}$ and $\mathbf{a} \in \mathcal{A}^{p}, 1 \leq p \leq \infty$. Also, for $\mathbf{c} \in \mathcal{A}^{p}$, let $S_{\mathbf{c}}(\cdot, \mathbf{a} ; \mathbf{t})$ be the unique $C^{2}$ function on the closure of $\mathcal{P}(\mathbf{c})$ that coincides with $S(\cdot, \mathbf{a} ; \mathbf{t})$ on $\mathcal{P}(\mathbf{c})$. For a point $x \in S^{1}$ and a sequence $\sigma=\left(\mathbf{a}_{0}, \mathbf{a}_{1}, \cdots, \mathbf{a}_{k}\right)$ of elements in $\mathcal{A}^{\infty}$, we will consider an affine map

$$
G_{x, \sigma}: \mathbb{R}^{m} \rightarrow \mathbb{R}^{k}
$$

defined by

$$
G_{x, \sigma}(\mathbf{t})=\left(\frac{d}{d x} S\left(x, \mathbf{a}_{i} ; \mathbf{t}\right)-\frac{d}{d x} S\left(x, \mathbf{a}_{0} ; \mathbf{t}\right)\right)_{i=1,2, \cdots, k} .
$$

Definition Let $0<\gamma \leq 1$ and $\delta>0$. A family $(21)$ is $(\gamma, \delta)$-generic at $x \in S^{1}$ if the following property holds: given $\mathbf{a}_{i} \in \mathcal{A}^{\infty}, 0 \leq i \leq d$, that have mutually distinct first alphabets $\left[\mathbf{a}_{i}\right]_{1}$, and given a positive integer $k<\gamma d$, we can choose a sequence $\sigma=\left(\mathbf{b}_{0}, \mathbf{b}_{1}, \cdots, \mathbf{b}_{k}\right)$ from $\mathbf{a}_{i}$ 's so that $\operatorname{Jac}\left(G_{x, \sigma}\right)>\delta$. Further, (21) is $(\gamma, \delta)$-generic on a subset $X \subset S^{1}$ if it is $(\gamma, \delta)$-generic at each point $x \in X$. Remark The linear part of $G_{x, \sigma}$ does not depend on the function $g$ but only on the functions $\varphi_{i}$ 's in the definition (20). Since the Jacobian $\operatorname{Jac}\left(G_{x, \sigma}\right)$ is defined as that of the linear part of $G_{x, \sigma}$, the $(\gamma, \delta)$-genericity above is a condition on the collection of functions $\varphi_{i}$ 's rather than that on the family $T_{\mathbf{t}}$ itself.

Assuming $(\gamma, \delta)$-genericity, we can give the following essential bound for the limit $\lim \sup _{q \rightarrow \infty} \mathbf{d}(q ; \mathbf{t})$.

Proposition 14 If a family (21) is $(\gamma, \delta)$-generic on $S^{1}$ for some $0<\gamma \leq 1$ and $\delta>0$, then, for any $\eta>0$,

$$
\operatorname{Leb}_{\mathbb{R}^{m}}\left\{\mathrm{t} \in \operatorname{Ball}\left(\eta ; \mathbb{R}^{m}\right) \mid \mathbf{d}(q ; \mathrm{t})>\gamma^{-1} \cdot\left(-\frac{2 \log \ell}{\log \lambda}+3\right)\right\}
$$

converges to 0 exponentially fast as $q \rightarrow \infty$. Especially,

$$
\limsup _{q \rightarrow \infty} \mathbf{d}(q ; \mathbf{t}) \leq \gamma^{-1} \cdot\left(-\frac{2 \log \ell}{\log \lambda}+3\right)
$$

holds for Lebesgue almost every $\mathbf{t} \in \mathbb{R}^{m}$.

Proof. Fix $\eta>0$. Put $N=[-2 \log \ell /(\log \lambda)]+2$,

$$
\alpha_{0}=\max _{\mathbf{t} \in \operatorname{Ball}\left(\eta ; \mathbb{R}^{m}\right)} \alpha(\mathrm{t})=(1-\lambda)^{-1} \max _{\mathbf{t} \in \operatorname{Ball}\left(\eta ; \mathbb{R}^{m}\right)}\left\|f_{\mathbf{t}}\right\|_{C^{2}}
$$

and

$$
p(q)=\left[\frac{\log \ell-\log \lambda}{\log \ell} \cdot q\right]+1
$$

Consider a parameter $\mathbf{t} \in \operatorname{Ball}\left(\eta ; \mathbb{R}^{m}\right)$ such that

$$
\mathbf{d}(q ; \mathbf{t})>\gamma^{-1}:\left(-\frac{2 \log \ell}{\log \lambda}+3\right) \text {. }
$$


Then

$$
\mathrm{d}\left(q, p(q) ; \lambda^{q} \alpha_{0}, \lambda^{q} \ell^{-q} \alpha_{0} ; \mathbf{t}\right)>\gamma^{-1} \cdot\left(-\frac{2 \log \ell}{\log \lambda}+3\right) \geq \gamma^{-1} N
$$

This implies that there exist $\mathbf{a}_{1}, \mathbf{a}_{2}, \cdots, \mathbf{a}_{d} \in \mathcal{A}^{q}$ and $\mathbf{c} \in \mathcal{A}^{p(q)}$ such that

- $d>\gamma^{-1} N$

- the first alphabets $\left[\mathbf{a}_{i}\right]_{1}, 1 \leq i \leq d$, are mutually distinct, and

- there exist points $x_{i j}, 1 \leq i<j \leq d$, in the closure of $\mathcal{P}(\mathbf{c})$ such that

$$
\left|\frac{d S_{\mathbf{c}}}{d x}\left(x_{i j}, \mathbf{a}_{i} ; \mathbf{t}\right)-\frac{d S_{\mathbf{c}}}{d x}\left(x_{i j}, \mathbf{a}_{j} ; \mathbf{t}\right)\right|<4 \lambda^{q} \ell^{-q} \alpha(\mathbf{t})+\lambda^{q} \ell^{-q} \alpha_{0} \leq 5 \lambda^{q} \ell^{-q} \alpha_{0} .
$$

Let $x_{\mathbf{c}} \in S^{\mathbf{1}}$ be the left endpoint of $\mathcal{P}(\mathbf{c})$. Then $x_{i j}-x_{\mathbf{c}}<\ell^{-p(q)}$ obviously. Since $\left|\frac{d^{2}}{d x^{2}} S\left(\cdot, \mathbf{a}_{i} ; \mathbf{t}\right)\right| \leq \alpha_{0}$ on the interior of $\mathcal{P}(\mathbf{c})$ from $(5)$, we have, for $1 \leq i<j \leq d$,

$$
\left|\frac{d S}{d x}\left(x_{\mathbf{c}}, \mathbf{a}_{i} ; \mathbf{t}\right)-\frac{d S}{d x}\left(x_{\mathbf{c}}, \mathbf{a}_{j} ; \mathbf{t}\right)\right|<5 \lambda^{q} \ell^{-q} \alpha_{0}+2 \ell^{-p(q)} \alpha_{0} .
$$

From the $(\gamma, \delta)$-genericity, we can choose a sequence $\sigma=\left(\mathbf{b}_{0}, \mathbf{b}_{1}, \cdots, \mathbf{b}_{N}\right)$ from $\left\{\mathrm{a}_{i}\right\}_{i=1}^{d}$ so that $\operatorname{Jac}\left(G_{x_{\mathrm{c}}, \sigma}\right)>\delta$.

Therefore, for each $\mathrm{t} \in \operatorname{Ball}\left(\eta ; \mathbb{R}^{m}\right)$ satisfying (26), there exist $\mathbf{c} \in \mathcal{A}^{p(q)}$ and a sequence $\sigma=\left(\mathbf{b}_{0}, \mathbf{b}_{1}, \cdots, \mathbf{b}_{N}\right)$ of elements in $\mathcal{A}^{q}$ such that

- $\operatorname{Jac}\left(G_{x_{c}, \sigma}\right)>\delta$ and

- $\mathrm{t} \in G_{x_{c}, \sigma}^{-1}\left([-\epsilon, \epsilon]^{N}\right)$ for $\epsilon=5 \lambda^{q} \ell^{-q} \alpha_{0}+2 \ell^{-p(q)} \alpha_{0} \leq 7 \lambda^{q} \ell^{-q} \alpha_{0}$.

For each of such $\mathbf{c}$ and $\sigma$, we have, by lemma 12 ,

$$
\mathbf{L e b}_{\mathbb{R}^{m}}\left(G_{x_{\mathrm{c}}, \sigma}^{-1}\left([-\epsilon, \epsilon]^{N}\right)\right) \leq \delta^{-1}(2 \epsilon)^{N} \cdot C \eta^{m-N}
$$

where $C$ is a constant that depends only on $m-N$. On the other hand, the number of combinations of $\mathbf{c}$ and $\mathbf{b}_{0}, \mathbf{b}_{1}, \cdots, \mathbf{b}_{N}$ is bounded by $\ell^{p(q)} \times \ell^{(N+1) q}$ obviously. Therefore we conclude that (25) is not larger than

$$
\delta^{-1}\left(14 \lambda^{q} \ell^{-q} \alpha_{0}\right)^{N} \cdot C \eta^{m-N} \cdot \ell^{p(q)+(N+1) q}<C \eta^{m-N} \delta^{-1}\left(14 \alpha_{0}\right)^{N} \ell\left(\ell^{2} \lambda^{N-1}\right)^{q} .
$$

Since $\ell^{2} \lambda^{N-1}<1$ from the definition of $N$, this is the claim of proposition 14 .

We close this section with a simple observation. Consider a sub-family of $T_{\mathbf{t}}$ that corresponds to a subset $\left\{\varphi_{\xi(j)}\right\}_{j=1}^{m^{\prime}}$ of the collection $\left\{\varphi_{i}\right\}_{i=1}^{m}$ of $C^{\infty}$ functions, that is, the family of maps defined by (20) and (21) with $\varphi_{i}, 1 \leq i \leq m$, replaced by $\varphi_{\xi(j)}, 1 \leq j \leq m^{\prime}$. Then the linear part of the map (24) defined for such subfamily is a restriction of the linear part of $G_{x, \sigma}$ to some linear subspace. Hence, from lemma 13 , wee see that, if such subfamily is $(\gamma, \delta)$-generic on a subset of $S^{1}$, the family $T_{\mathbf{t}}$ itself is $(\gamma, \delta)$-generic on that subset. 


\section{The proof of theorem 1}

Let $T_{\mathbf{t}}$ be a family of maps defined by (20) and (21) for some functions $g$ and $\varphi_{i}$, $1 \leq i \leq m$. Then the $n$-th iterate of $T_{\mathbf{t}}$ is written in the form

$$
T_{\mathrm{t}}^{n}(x, y)=\left(\ell^{n} x, \lambda^{n} y+f_{\mathrm{t}}^{(n)}(x)\right)
$$

where

$$
f_{\mathrm{t}}^{(n)}(x)=\sum_{j=0}^{n-1} \lambda^{n-j-1} g\left(\tau^{j}(x)\right)+\sum_{i=1}^{m} t_{i}\left(\sum_{j=0}^{n-1} \lambda^{n-j-1} \varphi_{i}\left(\tau^{j}(x)\right)\right) .
$$

If we put

$$
g^{(n)}(x)=\sum_{j=0}^{n-1} \lambda^{n-j-1} g\left(\tau^{j}(x)\right) \quad \text { and } \quad \varphi_{i}^{(n)}(x)=\sum_{j=0}^{n-1} \lambda^{n-j-1} \varphi_{i}\left(\tau^{j}(x)\right)
$$

the function $f_{\mathbf{t}}^{(n)}$ is written in the form

$$
f_{\mathbf{t}}^{(n)}(x)=g^{(n)}(x)+\sum_{i=1}^{m} t_{i} \varphi_{i}^{(n)}(x)
$$

Remark that (28) and (30) are completely in the same form as (20) and (21). Thus we can apply the argument in the previous sections to the family $T_{t}^{n}$ through the correspondence

$$
\ell^{n} \longleftrightarrow \ell, \quad \lambda^{n} \longleftrightarrow \lambda, \quad g^{(n)}(x) \longleftrightarrow g, \quad \varphi_{i}^{(n)}(x) \longleftrightarrow \varphi_{i}
$$

On the other hand, we can prove the following fact for the $n$-th iterate.

Proposition 15 For given $n \geq 1$, we can choose a finite collection of $C^{\infty}$. functions $\varphi_{i}, 1 \leq i \leq m$, so that the corresponding family $T_{\mathbf{t}}^{n}$, as (28) and (30), is. $(1 /(n+1), 1 / 2)$-generic on $S^{1}$, regardless of the $C^{2}$ function $g$.

Let us first see that theorem 1 follows from proposition 14 and proposition 15 . Since $\lambda \ell>1$, we can take a positive integer $n$ so large that

$$
(n+1)\left(-\frac{2 \log \ell^{n}}{\log \lambda^{n}}+3\right)=(n+1)\left(-\frac{2 \log \ell}{\log \lambda}+3\right)<\lambda^{n} \ell^{n} .
$$

For this $n$, we choose a collection of $C^{\infty}$ functions $\varphi_{i}, 1 \leq i \leq m$, as in proposition 15. This collection of functions satisfies the required condition in theorem 1 . In fact, let $g$ be an arbitrary $C^{2}$ function on $S^{1}$ and let $T_{\mathrm{t}}$ be the family of maps defined by (20) and (21). Then the family $T_{\mathbf{t}}^{n}$ of the $n$-th iterates is $(1 /(n+1), 1 / 2)$-generic from the claim of proposition 15. Apply proposition 14 to the family $T_{\mathrm{t}}^{n}$. Through the correspondence (31), the claim of proposition 14 and (32) imply that the map $T_{\mathbf{t}}^{n}$ satisfies the assumption of corollary 11 for 
Lebesgue almost every $\mathbf{t} \in \mathbb{R}^{m}$. Notice that the SBR measure for $T_{\mathbf{t}}^{n}$ is the same as that for $T_{\mathrm{t}}$. Hence the claim of corollary 11 implies that the pair $\left(\lambda, f_{\mathrm{t}}\right)$ belongs to the interior $\mathcal{D}^{\circ}$ of $\mathcal{D}$ for Lebesgue almost every $t \in \mathbb{R}^{m}$. This is nothing but the claim of theorem 1 .

Now our task is the proof of proposition 15. Actually proposition 15 is reduced to the following claim.

Proposition 16 For given $n \geq 1$ and $x \in S^{1}$, we can choose an open neighborhood $U_{x}$ of $x$ and a finite collection of $C^{\infty}$-functions $\varphi_{x, i}, 1 \leq i \leq m(x)$, so that the corresponding family $T_{\mathrm{t}}^{n}$, as (28) and (29) with $\varphi_{i}$ replaced by $\varphi_{x, i}$, is $(1 /(n+1), 1 / 2)$-generic on $U_{x}$, regardless of the $C^{2}$ function $g$.

In fact, if we assume proposition 16, we can choose a finite subset of $S^{\mathbf{1}}$, $\{x(1), x(2), \cdots, x(k)\}$, so that the neighborhoods $U_{x(j)}, 1 \leq j \leq k$, cover $S^{1}$. Let $\left\{\varphi_{i}\right\}_{i=1}^{m}$ be the union of the collection $\left\{\varphi_{x(j), i}\right\}_{i=1}^{m(x(j))}$ in proposition 16 over $1 \leq j \leq k$. Then consider the family $T_{t}^{n}$ defined by (20) and (21) for these $\varphi_{i}$ 's and an arbitrary $C^{2}$ function $g$. For each $1 \leq j \leq k$, the family of maps that is claimed to be $(1 /(n+1), 1 / 2)$-generic in proposition 16 for $x=x(j)$ is a subfamily of this family. Hence, as we observed in the last part of section 5 , this implies that the family $T_{\mathbf{t}}^{n}$ is $(1 /(n+1), 1 / 2)$-generic on each of the neighborhoods $U_{x(j)}, 1 \leq j \leq k$, and hence on whole $S^{1}$.

Finally we prove proposition 16 and finish the proof of theorem 1 .

Proof of proposition 16 We fix a positive integer $n$ and a point $x$ in $S^{1}$ below. The points $\mathbf{a}(x), \mathbf{a} \in \mathcal{A}^{n}$, are mutually distinct points in $\tau^{-n}(x)$. Notice that there is at most one element $\mathbf{a}$ in $\mathcal{A}^{n}$ such that the point $\mathbf{a}(x)$ is contained in the forward orbit of $x$ with respect to $\tau$. In fact, if $\mathrm{a}(x)=\tau^{q}(x)$ for some $q \geq 0$, the point $x$ should be periodic and $q \equiv(-n)$ modulo the period of $x$. But this implies the uniqueness of $\mathbf{a}(x)$ and hence that of $\mathbf{a}$.

For two elements $\mathbf{a}$ and $\mathbf{b}$ in $\mathcal{A}^{n}$, we denote $\mathbf{a} \prec \mathbf{b}$ if $\tau^{q}(\mathbf{b}(x))=\mathbf{a}(x)$ for some $q \geq \mathbf{0}$. If both $\mathbf{a} \prec \mathbf{b}$ and $\mathbf{b} \prec \mathbf{a}$ hold, we have $\mathbf{a}=\mathbf{b}$ because, otherwise, $\mathbf{a}(x)$ and $\mathbf{b}(x)$ would be contained in a periodic orbit that should pass through $x$, and hence contained in the forward orbit of $x$. Thus the relation $\prec$ is a partial order on $\mathcal{A}^{n}$. (The transitive and reflexive law are clear from the definition.)

For each $\mathbf{b} \in \mathcal{A}^{n}$, the number of elements $\mathbf{a} \in \mathcal{A}^{n}$ that satisfy $\mathbf{a} \prec \mathbf{b}$ is not larger than $n+1$. In fact, on the one hand, there is at most one element $\mathbf{a} \in \mathcal{A}^{n}$ such that $\mathbf{a}(x)=\tau^{q}(\mathbf{b}(x))$ for some $q \geq n$ because $\tau^{q}(\mathbf{b}(x))=\tau^{q-n}(x)$ belongs to the forward orbit of $x$. On the other hand, for each $0 \leq q<n$, there is at most one element $\mathbf{a} \in \mathcal{A}^{n}$ such that $\mathbf{a}(x)=\tau^{q}(\mathbf{b}(x))$. Thus, given a subset $Q$ of $\mathcal{A}^{n}$, at least one $(n+1)$-th of the elements are maximal in $Q$ with respect to the partial order $\prec$.

For $0<\epsilon<1 / 2$, let $U(\epsilon)$ be the $\epsilon$-neighborhood of $x$. For $\mathbf{a} \in \mathcal{A}^{n}$, let $U_{\mathbf{a}}(\epsilon)$ be the connected component of $\tau^{-n}(U(\epsilon))$ that contains $\mathbf{a}(x)$. In other words, $U_{\mathbf{a}}(\epsilon)$ is the $\ell^{-n} \epsilon$-neighborhood of $\mathbf{a}(x)$. Let $\nu>0$ be an integer constant that we will specify later. (We will give the concrete condition that $\nu$ should satisfy in (36).) For this $\nu$, we take $\epsilon_{0}>0$ so small that $\tau^{i}\left(U_{\mathrm{b}}\left(\epsilon_{0}\right)\right) \cap U_{\mathrm{a}}\left(\epsilon_{0}\right) \neq \emptyset$ for some $1 \leq i \leq n+\nu$ only if $\mathbf{a} \prec \mathbf{b}$. Then we take $C^{\infty}$-functions $\varphi_{\mathbf{a}}, \mathbf{a} \in \mathcal{A}^{n}$, on $S^{1}$ so that 
- the support of $\varphi_{\mathbf{a}}$ is contained in $U_{\mathbf{a}}\left(\epsilon_{0}\right)$,

- $\frac{d}{d x} \varphi_{\mathbf{a}}(x)=\lambda^{-n+1} \ell^{n}$ on $U_{\mathbf{a}}\left(\epsilon_{0} / 3\right)$, and

- $\left|\frac{d}{d x} \varphi_{\mathbf{a}}(x)\right|<2 \lambda^{-n+1} \ell^{n}$ on $S^{1}$.

Denote the collection of functions $\varphi_{\mathbf{a}}, \mathbf{a} \in \mathcal{A}^{n}$, by $\varphi_{x, i}, 1 \leq i \leq \ell^{n}$ and put $U_{x}=U\left(\epsilon_{0} / 3\right)$. We will show that this collection of functions and the open neighborhood $U_{x}$ satisfy the required condition in proposition 16, provided that we take the constant $\nu$ sufficiently large.

Henceforth we will consider the family of maps $T_{t}$ defined by (20) and (21) with $\varphi_{i}, 1 \leq i \leq m$, replaced by $\varphi_{\mathbf{a}}, \mathbf{a} \in \mathcal{A}^{n}$. We will put $g \equiv 0$ in (20) because the claim of proposition 16 is irrelevant to the choice of the $C^{2}$ function $g$. Our task is to check that the family $T_{t}^{n}$ is $(1 /(n+1), 1 / 2)$-generic on $U_{x}$.

Remark that, in considering the $n$-th iterate $T_{t}^{n}$ instead of $T_{t}$, we have to take the partition $\mathcal{P}^{n}:=\vee_{j=0}^{n-1} \tau^{-j}(\mathcal{P})$ and $\mathcal{B}=\mathcal{A}^{n}$ in the place of $\mathcal{P}$ and $\mathcal{A}$. For a word $\mathrm{b}=\left(b_{1}, b_{2}, \cdots,\right) \in \mathcal{B}^{\infty}$ on $\mathcal{B}$, the function that correspond to (23) is

$$
S^{(n)}(x, \mathbf{b} ; \mathbf{t}):=\sum_{j=1}^{\infty} \lambda^{j-1} f_{\mathrm{t}}^{(n)}\left([\mathbf{a}]_{j n}(x)\right)
$$

where $\mathbf{a}$ is the word on $\mathcal{A}$ that is obtained by appending $b_{1}, b_{2}, \cdots, \in \mathcal{A}^{n}$ in this order. But, from (20) and (29), we find

$$
S^{(n)}(x, \mathbf{b} ; \mathrm{t})=\sum_{j=1}^{\infty} \lambda^{j-1} f_{\mathrm{t}}\left([\mathbf{a}]_{j}(x)\right)=S(x, \mathbf{a} ; \mathbf{t})
$$

Hence, we can translate the $(\gamma, \delta)$-genericity of the $n$-th iteration $T_{\mathbf{t}}^{n}$ at a point $y \in S^{1}$ to the follwoing condition:

Condition: given $\mathbf{a}_{i} \in \mathcal{A}^{\infty}, 0 \leq i \leq d$, such that $\left[\mathbf{a}_{i}\right]_{n}$ are mutually distinct and given a positive integer $k<\gamma d$, we can choose a sequence $\sigma=$ $\left(\mathbf{a}_{\xi(0)}, \mathbf{a}_{\xi(1)}, \cdots, \mathbf{a}_{\xi(k)}\right)$ from $\mathbf{a}_{i}$ 's so that $\operatorname{Jac}\left(G_{y, \sigma}\right)>\delta$.

We will prove this translated condition for $y \in U_{x}$ with $\gamma=1 /(n+1)$ and $\delta=1 / 2$. Let $Q=\left\{\left[\mathbf{a}_{i}\right]_{n}\right\}_{i=1}^{d} \subset \mathcal{A}^{n}$. Then, from the observation above, at least one $(n+1)$-th of the elements of $Q$ are maximal in $Q$ with respect to the partial order $\prec$. Hence we can choose a sequence $\sigma=\left(\mathbf{a}_{\xi(0)}, \mathbf{a}_{\xi(1)}, \cdots, \mathbf{a}_{\xi(k)}\right)$ from $\mathbf{a}_{i}$ 's so that $\left[\mathrm{a}_{\xi(j)}\right]_{n}, 1 \leq j \leq k$, are maximal and mutually distinct in $Q$. We will prove $\operatorname{Jac}\left(G_{y, \sigma}\right)>1 / 2$ for $y \in U_{x}$. For $\mathbf{a} \in \mathcal{A}^{\infty}$ and $\mathbf{t} \in \mathbb{R}^{m}$, let us put

$$
S^{(1)}(x, \mathbf{a} ; \mathbf{t})=\sum_{i=1}^{n} \lambda^{i-1} f_{\mathbf{t}}\left([\mathbf{a}]_{i}(x)\right)
$$

and

$$
S^{(2)}(x, \mathbf{a} ; \mathbf{t})=\sum_{i=n+1}^{\infty} \lambda^{i-1} f_{\mathrm{t}}\left([\mathbf{a}]_{i}(x)\right)
$$


Also we define linear maps

$$
G_{y, \sigma}^{(\alpha)}: \mathbb{R}^{m} \rightarrow \mathbb{R}^{k}, \quad \alpha=1,2
$$

by

$$
G_{y, \sigma}^{(\alpha)}(\mathbf{t})=\left(\frac{d}{d x} S^{(\alpha)}\left(y, \mathbf{a}_{\xi(j)} ; \mathbf{t}\right)-\frac{d}{d x} S^{(\alpha)}\left(y, \mathbf{a}_{\xi(0)} ; \mathbf{t}\right)\right)_{j=1,2,3, \cdots, k}
$$

Obviously, $G_{y, \sigma}(\mathbf{t})=G_{y, \sigma}^{(1)}(\mathbf{t})+G_{y, \sigma}^{(2)}(\mathrm{t})$. From the choice of $\epsilon_{0}$ and $\sigma$, we have

$$
\tau^{q}\left(U_{\left[\mathbf{a}_{\xi(j)}\right]_{n}}\left(\epsilon_{0}\right)\right) \cap U_{\left[\mathbf{a}_{\xi\left(j^{\prime}\right)}\right]_{n}}\left(\epsilon_{0}\right)=\emptyset \quad \text { for } 1 \leq q \leq n+\nu
$$

if $0 \leq j \leq k$ and $0 \leq j^{\prime} \leq k$ are distinct. Hence, for any $\mathbf{a} \in \mathcal{A}^{\infty}$, the integers $q \geq 0$ for which $[\mathbf{a}]_{q}(y)$ belongs to the support of one of the functions $\varphi_{\left[\mathbf{a}_{\xi(j)}\right]_{n}}$, $0 \leq j \leq k$, are arranged at intervals larger than $n+\nu$.

Let $E_{0}$ be a $k$-dimensional linear subspace of $\mathbb{R}^{m}$ defined by

$$
E_{0}=\left\{\left(t_{1}, t_{2}, \cdots, t_{m}\right) \in \mathbb{R}^{m} \mid t_{i}=0 \text { if } i \neq \xi(j) \text { for any } 1 \leq j \leq k\right\} .
$$

Then, for $\mathbf{t}=\left(t_{1}, t_{2}, \cdots, t_{m}\right) \in E_{0}, y \in U_{x}$ and $1 \leq j \leq k$, we have

$$
S^{(1)}\left(y, \mathbf{a}_{\xi(j)} ; \mathbf{t}\right)=t_{\xi(j)} \lambda^{n-1} \varphi_{\left[\mathbf{a}_{\xi(j)}\right]_{n}}\left(\left[\mathbf{a}_{\xi(j)}\right]_{n}(y)\right)
$$

and, from the assumed properties of $\varphi_{\mathbf{a}}$ 's,

$$
\frac{d}{d x} S^{(1)}\left(y, \mathbf{a}_{\xi(j)} ; \mathbf{t}\right)=t_{\xi(j)}
$$

On the other hand, we have, for $\mathbf{t} \in E_{0}$ and $y \in U_{x}$,

$$
S^{(1)}\left(y, \mathbf{a}_{\xi(0)} ; \mathbf{t}\right)=\frac{d}{d x} S^{(1)}\left(y, \mathbf{a}_{\xi(0)} ; \mathbf{t}\right)=0 .
$$

Hence the matrix corresponding to $\left.G_{y, \sigma}^{(1)}\right|_{E_{0}}: E_{0} \rightarrow \mathbb{R}^{k}$ is the unit matrix of size $k$ if we set the order of coordinate axes of $E_{0}$ appropriately.

On the other hand, the first $n+\nu$ terms in the sum on the right hand side of (34) is zero when $\mathbf{t} \in E_{0}$. Thus, for $\mathbf{t} \in E_{0}$, the function $S^{(2)}\left(y ; \mathbf{a}_{\xi(j)}\right.$; $\left.\mathbf{t}\right)$ is written in the form

$$
S^{(2)}\left(y, \mathbf{a}_{\xi(j)} ; \mathbf{t}\right)=\sum_{i=2 n+\nu+1}^{\infty} \sum_{p=1}^{k} t_{\xi(p)} \lambda^{i-1} \varphi_{\left[\mathbf{a}_{\xi(p)}\right]_{n}}\left(\left[\mathbf{a}_{\xi(j)}\right]_{i}(y)\right) .
$$

The absolute value of the coefficient of $t_{\xi(j)}$ in $\frac{d}{d x} S^{(2)}\left(x, \mathbf{a}_{\xi(j)} ; \mathbf{t}\right)$ is smaller than

$$
\sum_{i=2 n+\nu+1}^{\infty} \lambda^{i-1} \ell^{-i} \cdot 2 \ell^{n} \lambda^{-n+1} \leq 2 \lambda^{n+\nu} \ell^{-n-\nu}(1-\lambda / \ell)^{-1}
$$


Hence, each entry of the matrix corresponding to $\left.G_{y, \sigma}^{(2)}\right|_{E_{0}}: E_{0} \rightarrow \mathbb{R}^{k}$ is smaller than $4 \lambda^{n+\nu} \ell^{-n-\nu}(1-\lambda / \ell)^{-1}$ and hence its operator norm is not larger than $4 k \lambda^{n+\nu} \ell^{-n-\nu}(1-\lambda / \ell)^{-1}$.

Remark that we have $k \leq \ell^{n} /(n+1)<\ell^{n}$. So if we take $\nu$ so large that

$$
\left(1-4 \lambda^{n+\nu} \ell^{-\nu}(1-\lambda / \ell)^{-1}\right)^{\ell^{n}}>1 / 2
$$

we can conclude

$$
\operatorname{Jac}\left(G_{y, \sigma}\right) \geq \operatorname{Jac}\left(\left.G_{y, \sigma}\right|_{E_{0}}\right)>1 / 2 \quad \text { for } y \in U_{x}
$$

using lemma 13.

\section{Appendix}

We prove the facts given in Examples 1 and 2. Let us first consider Example 1. In this case, $\ell=2$ and $\mathcal{A}=\{0,1\}$. We claim that $\mathbf{e}(1)=1$. Obviously this claim and proposition 8 show the absolutely continuity of the SBR measures. Let us consider two element $\mathbf{a}$ and $\mathbf{b}$ in $\mathcal{A}^{\infty}$ such that $[\mathbf{a}]_{1}=0$ and $[\mathbf{b}]_{1}=1$. For $|x| \leq 2 / 5$, we can check (actually, using a calculator) that

$$
\begin{aligned}
& \left|\frac{d}{d x} S(x, \mathbf{a})-\frac{d}{d x} S(x, \mathbf{b})\right| \\
& =\left|\frac{1}{2} \cos \left([\mathbf{a}]_{1}(x)\right)-\frac{1}{2} \cos \left([\mathbf{b}]_{1}(x)\right)\right|+\mid \frac{\lambda}{4} \cos \left([\mathbf{a}]_{2}(x)-\frac{\lambda}{4} \cos \left([\mathbf{b}]_{2}(x) \mid+\cdots\right.\right. \\
& >2 \cdot \frac{1}{2} \cos \frac{2 \pi}{5}-\frac{\lambda}{4}\left(\cos \frac{\pi}{5}+\cos \left(\frac{\pi}{2}-\frac{\pi}{5}\right)\right)-\frac{\lambda^{2}}{4} \frac{1}{1-\lambda / 2}>0 .
\end{aligned}
$$

In the case $2 / 5 \leq x \leq 1-2 / 5$, each of the points $[\mathbf{a}]_{2}(x)$ and $[\mathbf{b}]_{2}(x)$ is contained in the $1 / 40$ neighborhood of either of $k / 8, k=1,3,5,7$. Hence

$$
|S(x, \mathbf{a})-S(x, \mathbf{b})|>2\left\{\sin (2 \pi / 5)-\lambda \sin (3 \pi / 10)-\lambda^{2}(1-\lambda)^{-1}\right\}>0 .
$$

These imply $\mathbf{e}(1)=1$.

The proof of the facts in Example 2 is easy. We can check that the subset $\operatorname{graph}(\varphi)$ is forward invariant and the map $\Psi$ in section 2 is written as $\Psi(x, \mathbf{a})=$ $(x, \varphi(x))$. Hence the SBR measure $\mu=\Psi(\mathbf{m} \times \nu)$ is supported on $\operatorname{graph}(\varphi)$. and totally singular.

\section{References}

[1] J.C.Alexander \& J.A. Yorke, Fat Baker's transformations, Ergodic theory and dynamical systems, 4, 1-23 (1984)

[2] P. Erdös, On a family of symmetric Bernoulli convolutions, Amer. J. Math. 61, 974-976, (1939) 
[3] B. Solomyak, On the random series $\sum \pm \lambda^{n}$ (an Erdös problem), Ann. of Math. (2) 142, no. 3, 611-625, (1995)

[4] B.Solomyak \& Y. Peres, Absolute continuity of Bernoulli convolutions, a simple proof, Math. Research Letters 3, 231-239 (1996)

[5] M. Tsujii, A measure on the space of smooth mappings and dynamical system theory., J. Math. Soc. Japan 44, no. 3, 415-425, (1992)

[6] B. R.Hunt, T. Sauer \& J. A. Yorke, Prevalence: a translation-invariant "almost every" on infinite-dimensional spaces, Bull. Amer. Math. Soc. , 27, no.2, 217-238(1992); Addendum ibid. 28 , no. 2, 306-307, (1993) 